\title{
Prácticas de consumo alimentario de familias desplazadas por el conflicto armado, asentadas en Bosa, Bogotá*
}

\author{
Food Consumption Practices of Families Displaced \\ by the Armed Conflict living in Bosa, Bogota
}

Myriam Carmenza Sierra-Puentes ${ }^{* *}$ ORCID: 0000-0002-9829-2827

Angie Marcela Bejarano Riveros ORCID: 0000-0001-9483-920X

Fundación Universitaria Konrad Lorenz

Recibido: 22 de junio de 2019

Revisado: 7 de agosto de 2019 Aceptado: 2 de noviembre 2019

\section{Resumen}

Colombia es el país con mayor número de desplazados internos a nivel mundial. La mayoría de las victimas provienen de zonas rurales. Este estudio tuvo como objetivo indagar y describir las prácticas de consumo alimentario actuales y previas al desplazamiento forzado. Por medio de una metodología cualitativa se analizó y teorizó el discurso de cinco mujeres víctimas de desplazamiento forzado asentadas con sus familias en la localidad de Bosa, Bogotá. Por medio de una entrevista semiestructurada se recolectó la información y fue analizada con el software $\mathrm{N}$-vivo11, siguiendo el análisis de la teoría fundamentada. Los hallazgos revelan que las prácticas de consumo alimentario previas al desplazamiento, se caracterizaron por la obtención de víveres a través de labores agropecuarias como la siembra y se consumían mínimo tres comidas durante el día. Actualmente, la cantidad de las raciones de consumo diarias han disminuido, así como la frecuencia de compra y las fuentes de obtención de la alimentación. En conclusión, las prácticas de consumo alimentario se han transformado, lo cual ha obligado a las familias a cambiar su dieta por una más precaria y de baja calidad.

Palabras clave: prácticas de consumo; consumo alimentario; desplazamiento; alimentación; consumo familiar.

Citar como: Sierra-Puentes, M. \& Bejarano R., A. (2020). Prácticas de consumo alimentario de familias desplazadas por el conflicto armado, asentadas en Bosa, Bogotá. Diversitas: Perspectivas en psicología, 16(1), 143-155. Dol: https://doi.org/10.15332/22563067.5547

Correspondencia: Myriam C. Sierra Puentes, Facultad de Psicología, Fundación Universitaria Konrad Lorenz, Carrera 9BIS \# 62-43, Bogotá, Colombia. Correo electrónico: myriamc.sierrap@konradlorenz.edu.co.

Agradecimientos: la investigación presentada en este artículo fue financiada por la Fundación Universitaria Konrad Lorenz de Colombia. Contribuciones de autor: las dos autoras contribuyeron a todas las etapas de la revisión. Igualmente, aprobaron la versión final del manuscrito. 


\section{Abstract}

Colombia is the country with the highest number of people displaced by violence in the world. Most of the victims come from rural areas. The aim of this study is to survey and describe the current food consumption habits of families as they compare to those they had before their forced displacement. Employing a qualitative methodology, the statements of five women victims of forced displacement who settled with their families in Bosa, Bogotá, were analyzed and theorized. Through a semi-structured interview, the information was collected and analyzed with the N-vivo 11 software, following the grounded theory analysis. The findings reveal that the food consumption habits before displacement are characterized by the gathering of food through agricultural practices like crop growing and by the consumption of a minimum of three meals a day. Nowadays, the portion size of the daily meals has diminished, together with the grocery purchase frequency and the sources of food. In conclusion, food consumption practices have been transformed, which has forced families to change their diet to one more precarious and of poorer quality.

Keywords: consumption practices; food consumption; displacement; food; family consumption.

\section{Introducción}

Colombia ha vivido un conflicto armado durante más de 50 años, unido a este flagelo se encuentra el incremento de las desigualdades económicas, situaciones que han deteriorado las condiciones de vida de la población en general, especialmente de las personas afectadas directamente por la guerra, poblaciones desplazadas y comunidades receptoras. En la actualidad, debido a las guerras civiles, el fenómeno del desplazamiento interno se ha masificado, generando crisis migratorias similares a las ocurridas durante la segunda guerra mundial (United Nations Office for the Coordination of Humanitarian Affairs, 2015).

En el 2015, el informe del Internal Displacement Monotoring Center and Norwegian Refugee Council (IDMC \& NRC, 2017) con una cifra de 6’270.000 posicionó a Colombia como el segundo país con mayor cantidad de desplazados por la violencia a nivel mundial, y el primero en el continente americano. En el más reciente informe del IDMC, Colombia se establece como el país con mayor cantidad de desplazados por conflictos a nivel mundial con una cifra de 7’246.000 víctimas registradas hasta el
31 de diciembre del 2016 (IDMC \& NRC, 2017). De acuerdo con el boletín de la Consultoría para los Derechos Humanos y el Desplazamiento (CODHES, 2016), durante el año 2015 aproximadamente 15.495 personas fueron víctimas de desplazamiento forzado. Las cifras previas, permiten deducir que el desplazamiento forzado a partir de hechos violentos continúa siendo una problemática de impacto para el país.

Durante el año 2016, se llegó a un acuerdo final entre el gobierno y las Fuerzas Armadas Revolucionarias de Colombia (FARC), dando fin a más de 50 años de conflicto, pretendiendo así llegar a una etapa de postconflicto, en dónde, se disminuyera la violencia en el país y se implementaran los puntos establecidos en el acuerdo. A pesar de esto, se siguen reportando hechos de violencia y despojo en Colombia, por lo que se han registrado asesinatos de líderes sociales y nuevos desplazamientos (IDMC \& NRC 2017). Por lo que, pese al cese del conflicto, se continúan registrando víctimas de desplazamiento forzado.

Con el fin de restituir los derechos de las víctimas, se instauró la Ley 1448 de 2011, a través de esta, se han reestablecido los derechos de más de 500.000 
personas. No obstante, durante la evaluación de medidas integrales de reparación realizada por The Carr Center for Human Righst Policy y The Harvard Humanitarian Initiative, se estableció que se ha reparado menos del $10 \%$ de la población afectada, por lo que el sistema de reparación tiene recursos insuficientes para suplir las necesidades en su totalidad, incumpliendo con las condiciones establecidas en la ley de reparación (CARR \& HIN 2015). Es así como las víctimas se ven vulneradas y desprotegidas por el estado, llevándolos a vivir condiciones de pobreza y revictimización.

Los desplazados forzados conforman uno de los grupos con mayor vulnerabilidad en el territorio colombiano (Hernández-Bello \& Gutiérrez-Bonilla, 2008), porque las familias se enfrentan a situaciones como: pérdidas económicas; la descomposición del núcleo familiar; la deserción escolar; el asentamiento en condiciones precarias y la insatisfacción de necesidades básicas a nivel alimentario y de salud (Aysa-Lastra, 2011; Bozzoli, Bruck \& Wald, 2012; Carrillo, 2009; Espinosa-Menéndez, Galindo-Rosero, Bastidas-Lopera, \& Monsalve-Rojas, 2012; Ibáñez \& Moya, 2006; Macuacé, 2009; Muggah, 2000). De esta forma, la población desplazada se encuentra viviendo por debajo del umbral de pobreza o incluso de la indigencia, llegando a considerarse que los hogares desplazados mantienen un estilo y calidad de vida más precaria en relación con hogares pobres urbanos, afectando así, el cubrimiento de necesidades básicas y el óptimo desarrollo.

Con relación a la alimentación, países como España han adelantado estudios con población migrante, en donde, los principales hallazgos dejan en evidencian la presencia de desnutrición y desórdenes alimentarios como obesidad, ocasionados generalmente por privación de alimentos, malos hábitos alimentarios y cambios en las costumbres (Custodio, Ortiz, \& Rodríguez, 2014; Marín, Gutiérrez, Guallar, Banegas, Regidor, \& Rodríguez, 2010; Menal, Fajó, \& Marques, 2011; Miguel, Cruz, Masvidal, Frutos, Estabanell, \& Riera 2012; Oliván, 2000, 2004).

En Colombia, la Encuesta Nacional de la Situación Nutricional (Instituto Colombiano de Bienestar Familiar (ICBF), 2010) revela que $13.2 \%$ de la población infantil padece de retraso en el crecimiento, el índice de desnutrición global es de $3.4 \%$, las condiciones de desnutrición se asocian a mayor cantidad de integrantes en la familia y situación socioeconómica, denotando que la inseguridad alimentaria se da principalmente por la carencia de recursos económicos para obtener alimentos. A pesar de que el índice nacional de desnutrición disminuyó, el sobrepeso aumentó, el $51.2 \%$ de la población entre los 18 y 64 años padece esta patología, además, dicha condición tiende a presentarse con mayor frecuencia en zonas urbanas, evidenciando que la alimentación de las grandes ciudades no es nutritiva.

En cuanto al estado alimenticio de la población desplazada, existen estados alarmantes de desnutrición en menores de edad, ya que hay modificaciones en la dieta alimentaria asociadas a los escasos ingresos económicos en el hogar (Gamboa, López, Vera, \& Prada, 2007; Machado, Calderón, \& Machado, 2014). Al presentarse dificultades en la alimentación, se aumenta la probabilidad de desencadenar desórdenes alimentarios como la obesidad, desnutrición y el desarrollo de enfermedades como la malaria, y parásitos intestinales en niños y adolescentes (Carmona, 2011; Instituto Colombiano de Bienestar Familiar, 2010; Gómez et al., 2012).

Por otra parte, el estudio de Prada, Herrán y Ortiz (2008) realizado en Santander, determinó que un $34.7 \%$ de las familias desplazadas no tienen acceso a la compra de alimentos debido a la carencia de dinero. Además, el 95\% mantiene inseguridad alimentaria, ya que no consumen comidas inocuas y nutritivas. De igual forma, la población migrante por la violencia mantiene una dieta alimentaria inadecuada, constatando casos de desnutrición y baja calidad de vida (Prada et al., 2010). Las prácticas de consumo alimentario de la población desplazada se ven modificadas desfavorablemente, por lo que la nutrición y la seguridad alimentaria se ven vulneradas. En el informe del Programa Mundial de Alimentos (Programa Mundial de Alimentos, 2005) sobre el estado nutricional, de alimentación y condiciones de salud de la población desplazada en Colombia, se identifica que el $\mathbf{8 8 \%}$ de las familias desplazadas han disminuido el número de comidas debido a la falta de recursos económicos. Además, el consumo de proteínas necesarias para una dieta sana se ha visto reducido, por lo que en promedio 
las familias consumen alimentos ricos en proteínas una vez a la semana, dando prioridad a la ingesta de alimentos calóricos. Es así como el derecho a la alimentación se ve vulnerado y surge la necesidad de implementar políticas y estudios que orienten el progreso en el déficit alimentario de la población.

Por otra parte, según datos recopilados por el Centro Nacional de Memoria Histórica (2013), son los hombres en su mayoría, las víctimas de desaparición forzada y homicidio, de forma tal que sobre las mujeres recaen otros hechos victimizantes asociados a la condición de género, en los que se destacan las amenazas y el desplazamiento forzado. Los datos del Registro Único de Víctimas (RUv, 2017) al 11 julio de 2017, registran que desde el año 1985 hasta el 2017, las mujeres son principalmente víctimas de despojo forzado de tierras, delitos contra la libertad e integridad sexual, amenazas y desplazamiento. En contraste, los hombres son mayoritariamente víctimas de homicidio, desaparición forzada, secuestro, tortura y minas antipersonales. Se han registrado 3'661.777 casos de mujeres víctimas de migración forzada, cifra superior a los 3'477.974 de hombres que han declarado padecer este hecho Registro Único de Victimas (RUV, 2017).

Las mujeres víctimas del desplazamiento, mantienen necesidades particulares de protección y asistencia que superan las de los hombres, además, estas, ejecutan prácticas de supervivencia de mayor eficacia en relación con el género masculino (Lakshman, 2011). Estudios nacionales, revelan que las mujeres desplazadas poseen antecedentes culturales de violencia doméstica, uniones maritales tempranas, número elevado de hijos y escasa escolaridad (Pareja \& lañez, 2014). Así, al enfrentarse a las demandas laborales de la ciudad, las mujeres asumen la jefatura del hogar, además de desarrollar alteraciones en la salud física y mental, surgen también dificultades para adquirir alimentos ocasionando pérdida de peso y vulnerabilidad para adquirir patologías (Mogollón \& Vásquez, 2006). Por lo anterior, son las mujeres las que con mayor frecuencia son desplazadas y deben asumir en las ciudades nuevos roles sociales, afrontando la jefatura del hogar y ejerciendo nuevas labores que les permitan sustentar la economía familiar, realizando modificaciones en el estilo de vida. En el caso de la alimentación, las mujeres se ven obligadas a adaptar la dieta familiar a partir de las condiciones económicas.

Como resultado de la modificación del estilo de vida, las personas migran a ciudades dónde puedan rehacer su vida. Bogotá se ha convertido en la ciudad principal de asentamiento de víctimas, los barrios periféricos en localidades como Bosa, Rafael Uribe, Ciudad Bolívar, Usme y San Cristóbal son amplios receptores de personas desplazadas que escapan de la guerra. Sin embargo, no encuentran condiciones de vida adecuadas, por lo que se instauran revictimizaciones y se evidencian falencias en el cumplimiento de las políticas públicas (CODHES, 2014).

A partir de la revisión teórica previa y la escasa información sobre la alimentación de población desplazada, surge el interés en identificar las prácticas de consumo alimentario de la población desplazada por el conflicto asentada en la localidad de BosaBogotá, con el fin de esclarecer las condiciones alimentarias y la modificación en la dieta que han tenido que realizar las familias para adaptarse a su nuevo estilo de vida.

\section{Método}

El objetivo principal de la investigación fue reconocer y describir las prácticas de consumo actual y previo al desplazamiento, de las familias víctimas del conflicto armado nacional asentadas en la localidad de Bosa. Para el diseño del método del presente estudio se tuvieron en cuenta los parámetros en investigación cualitativa postulados por Twinning y Heller (2017). El estudio utilizó un enfoque cualitativo, se analizó la información a través de la teoría fundamentada y se organizaron los datos en categorías. Por medio de un muestreo por conveniencia, se seleccionaron las participantes, las cuales cumplían con los siguientes criterios de selección: a) haber sido víctima de desplazamiento forzado, b) ser mujer y c) estar a cargo de la alimentación familiar antes del desplazamiento y en la actualidad.

La categoría orientadora central fue la alimentación, durante el desarrollo del estudio, bajo el método fenomenológico, se diseñó una guía de entrevista 
semiestructurada, la cual contó con una validación previa por jurados expertos. La guía evaluó los ejes de indagación relacionados con las prácticas de consumo alimentario actual y previo al desplazamiento. Se contó con la participación de cinco (5) mujeres víctimas del conflicto armado, desplazadas y asentadas con sus familias en la localidad de Bosa, Bogotá. Cada una de las participantes firmó un consentimiento informado en dónde se expusieron los fines del estudio y la opción de retirarse de la investigación en cualquier momento. Se aplicó la entrevista en terreno y fue grabada con un dispositivo electrónico. Los datos fueron transcritos $y$ analizados posteriormente con el software $\mathrm{N}$-vivo 11.

\section{Resultados}

La tabla 1 se presenta la composición de las comidas antes del desplazamiento y actualmente. En las categorías detectadas, se describe la diversidad de alimentos presentes en cada una de las comidas. Respecto a la alimentación actual, es notorio el remplazo de alimentos nutritivos como proteínas, por aquellos que para los entrevistados generan saciedad como los granos, las harinas y los tubérculos. Es destacable el hecho que, en la alimentación previa al desplazamiento, se consumieran tres proteínas en el día, además, la variedad de comestibles es notoria.

Tabla 1.

Composición de comidas diarias antes y ahora.

\begin{tabular}{lll}
\hline \multicolumn{1}{c}{ Comidas Diarias } & \multicolumn{1}{c}{ Antes } & \multicolumn{1}{c}{ Ahora } \\
\hline Desayuno & $\begin{array}{l}\text { Huevo, lácteo, pescado, cereal, carne de res, } \\
\text { cerdo, caldo, tubérculos y harinas }\end{array}$ & $\begin{array}{l}\text { Agua de panela, cereal, lácteo, } \\
\text { huevo, harina y café. }\end{array}$ \\
\hline \multirow{2}{*}{ Almuerzo } & $\begin{array}{l}\text { Carne de res, carne de cerdo, granos, harinas, } \\
\text { tubérculos, huesos, jugo natural, lácteo, sopa, } \\
\text { langosta, camarones, pescado, verdura }\end{array}$ & $\begin{array}{l}\text { Embutidos, carne, menudencias, } \\
\text { huevo, granos, harinas, tubérculos, } \\
\text { sopa y verdura. }\end{array}$ \\
\hline Cena & $\begin{array}{l}\text { Carne de res, pescado, cereal, harina, tubérculos, } \\
\text { lácteo, sopa, jugo natural }\end{array}$ & Café y harina. \\
\hline
\end{tabular}

Fuente: elaboración propia.

En los discursos de las entrevistadas se identifica la variedad de alimentos en la dieta diaria antes del desplazamiento como se observa en la tabla 2, la diversidad de proteínas animales, junto con la presencia de harinas y tubérculos, caracterizaban la composición diaria de las meriendas, es notable la

Tabla 2.

Consumo de alimentos antes del desplazamiento gran variedad de fuentes de proteína, por lo que el consumo de carnes se posiciona como un alimento indispensable en las tres comidas básicas diarias de las familias entrevistadas, según los discursos de las participantes.

\begin{tabular}{|c|c|c|}
\hline \multirow{8}{*}{$\begin{array}{l}\text { Alimentos } \\
\text { antes del } \\
\text { desplazamiento }\end{array}$} & Carnes & $\begin{array}{l}\text { Pollo, piangua, mariscos, langostas, carne de cerdo, carne de } \\
\text { guagua, gallina, carne de ñeque, carne de jabalí, carne de raya, } \\
\text { carne de conejo, carne de paloma, carne de res, camarones, } \\
\text { pescado, tortuga y carne de ponce. }\end{array}$ \\
\hline & Cereales & Panes, arroz, trigo. \\
\hline & Verduras & Verduras de huerta. \\
\hline & Harinas y tubérculos & Yuca, papa y plátano. \\
\hline & Frutas & Frutas de cosecha, jugos naturales. \\
\hline & Granos & Frijoles, lentejas, garbanzos. \\
\hline & Huevos y lácteos & Huevos de gallina criolla, queso, leche. \\
\hline & Bebidas carbonatadas & Gaseosas, jugos en polvo. \\
\hline
\end{tabular}

Fuente: elaboración propia. 
Así pues, el reporte de los alimentos se suma a las narraciones de las mujeres, en donde se destaca la abundancia y diversidad de alimentos en el consumo diario como se identifica en los relatos de las participantes de este estudio: "allá comíamos bueno, tapa de pescado, o sino en el almuerzo sancocho de pescado o sudado de piangua, o mariscos así como camarones, langostas, eso era lo que comíamos allá, y en la merienda pescado frito con plátano y arroz". ... "El desayuno que se respete es ese, o frita cerdo, o frita carne con yuca también, o hace un plátano frito, o plátano cocido, o come con bollo de mazorca con queso y sueros, pero ese es un desayuno, para allá, para nosotros, ese es un desayuno, no pan".
Es relevante resaltar que, en comparación al consumo actual, presentado en la tabla 3, la ingesta de proteínas se reduce, así como la variedad de estas, dando prioridad al consumo de carnes de fácil acceso económico como lo son las menudencias y los embutidos. Por lo anterior, las familias deben adaptarse al entorno y adquirir alimentos por medio de la compra, por lo que el factor económico se convierte en un requerimiento indispensable para acceder al consumo, dicha cuestión surge como resultado de las dificultades de empleabilidad en la ciudad y el incumplimiento de la reparación por parte del estado.

Tabla 3.

Consumo actual de alimentos.

\begin{tabular}{lll}
\hline & \multicolumn{1}{l}{ Carnes } & Pollo, vísceras, huesos, carne molida, menudencias, embutidos. \\
\cline { 2 - 3 } & Cereales & Pan. \\
\cline { 2 - 3 } Alimentos actuales & Verduras & Verduras de conteiner de abastos ${ }^{1}$ \\
\cline { 2 - 3 } & Harinas y tubérculos & Arroz, papas, plátano. \\
\cline { 2 - 3 } & Frutas & Frutas de cosecha. \\
\cline { 2 - 3 } & Granos & Frijoles, lentejas. \\
\hline Huevos y lácteos & Leche en polvo, huevos comprados. \\
\hline
\end{tabular}

Fuente: elaboración propia.

El consumo de alimentos se ve modificado, de forma que con el desplazamiento, las familias incursionan a probar y buscar nuevos productos que se ajusten a sus necesidades económicas, "las menudencias, que es lo que yo más compro, menudencia, porque es lo más barato". Inclusive en ocasiones, la carencia de recursos económicos impide el acceso a alimentos. La modificación abrupta de la alimentación, deteriora el estado de salud de las familias, principalmente, por la disminución de las comidas diarias y la ausencia de proteínas en estas; "nosotros ahorita prácticamente, hacemos una sola comida, ósea, con eso de pronto, tratamos como de nivelarlo"... "Yo no como carne, nosotros comemos carne de vez en cuando".

1 Se denomina abastos a una plaza de mercado en la ciudad de Bogotá, en la cual se comercializan todo tipo de productos perecederos.
En la Figura 1, se aprecian las diversas fuentes de origen de los alimentos antes del desplazamiento y en la actualidad. Es notorio que previo al desplazamiento, las familias obtenían la mayoría de los víveres, a través de labores agropecuarias como la siembra, la cría de animales, la caza y la pesca, por lo cual, el dinero no era una variable indispensable para tener acceso a proteínas de origen animal, frutas, verduras, cereales, harinas y tubérculos, en el entorno rural se facilitaba el acceso a una dieta balanceada y nutritiva ... "por allá se siembra todo eso, el ñame, todo eso se siembra y por ejemplo, allá también se acostumbra mucho a sembrar lo que es, lo que llaman aquí habichuela, allá uno le dice frijol, siembra uno frijol, cabecita negras así, pimentón por allá en esa tierra se siembra mucho". 


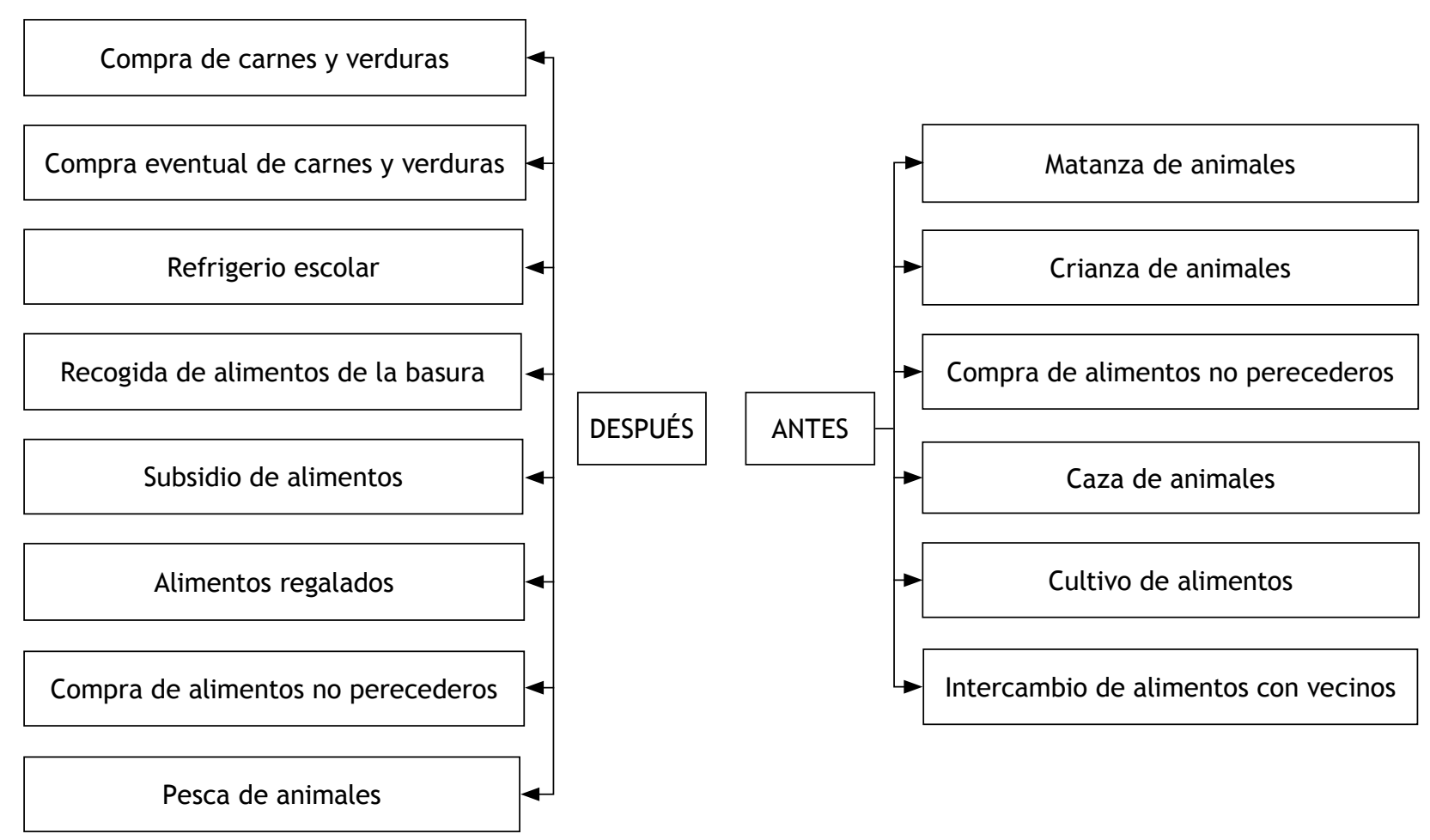

Figura 1. Obtención de alimentos antes y ahora. Fuente: elaboración propia.

Además, las prácticas culturales del entorno, facilitaban la adquisición de alimentos, debido a que las familias sostenían relaciones sociales con vecinos, compartiendo e intercambiando alimentos, disponiendo de seguridad alimentaria "usted va donde la vecina y le llevó, o la vecina trajo un spaghetti o el cambiazo que dicen que por allá se cambia mucho la comida, que vecina, yo hice esto, pero no me gusta, usted ¿qué hizo? Cambiemos"

Como resultado de la adaptación al entorno, en la actualidad, la mayoría de los alimentos provienen de la compra de estos, en diversos establecimientos como plazas de mercado, supermercados, tiendas de barrio o graneros. Esto unido a los subsidios del
Estado, del colegio o de personas naturales, favorecen la dieta de las familias. No obstante, debido a la carencia de fuentes económicas, las madres de los hogares se someten a la búsqueda de alimentos en contenedores de basura, de esta forma, adquieren frutas y verduras gratuitamente, supliendo la alimentación que demandan los integrantes de la familia, ..."una señora nos presta un carrito y vamos con el carrito y en los conteiner de afuera donde depositan lo que dice uno basura, pero ahí consigue uno cosas buenas, ósea, yo digo que si la gente que está bien que tiene plata lo mira como un desecho, sí, puede que sí porque están ya en container, pero entonces uno va y escoge y son cosas que, al día de hoy, no nos ha hecho daño". 
Tabla 4.

Prácticas de consumo alimentario previas y actuales.

\begin{tabular}{|c|c|}
\hline \multicolumn{2}{|c|}{ Prácticas de Consumo } \\
\hline Previas & Actuales \\
\hline Uso de pipeta & Reducción del número de comidas diarias \\
\hline Preparación de comida en leña & Consumo de nuevos alimentos \\
\hline Toma de alimentos de cultivo & Ausencia de proteína \\
\hline Horarios determinados para alimentarse & Nuevas formas de preparar alimentos \\
\hline Preparación de comida especial en celebraciones & Preparación variada del mismo alimento \\
\hline Compartir alimentos con familia y vecinos & $\begin{array}{l}\text { Compra de alimentos para preparar comida en celebra- } \\
\text { ciones }\end{array}$ \\
\hline Conservación de alimentos en cavas & Recoger alimentos en containers de abastos \\
\hline Preservación de carne con sal & Ausencia de compra de verduras \\
\hline Matanza de animales para obtener carnes & Uso de estufa a gas \\
\hline Caza de animales & Almuerzo en el colegio \\
\hline Pesca de animales & Carencia de horarios para comer \\
\hline \multirow[t]{7}{*}{ Cultivar alimentos } & Disminución de celebraciones \\
\hline & Compra frecuente de alimentos \\
\hline & Reservar alimentos para no cocinar diariamente \\
\hline & Búsqueda de bajos precios al comprar \\
\hline & Preparación de una sola comida \\
\hline & Empacar alimentos para llevar \\
\hline & Uso de hielo para conservar alimentos \\
\hline
\end{tabular}

Fuente: elaboración propia.

Como se aprecia en la Tabla 1, dentro de las prácticas de consumo alimentario previas al desplazamiento, es común la preparación de alimentos en fogones de leña, el uso de pipetas de gas, la toma de verduras y tubérculos de huertos o cultivos, el consumo de animales para festejar alguna celebración importante, el compartir familiar a la hora de comer y la conservación de alimentos perecederos por medio de métodos tradicionales. La comida permite generar enlaces y afianzar las relaciones, debido a que la familia comparte durante la preparación y el consumo de los alimentos. ... "en diciembre se acostumbraba a hacer lo que es tamales, pasteles que le llaman allá. Matar chivo o carnos, en diciembre, sí, también nos reuníamos y hacíamos varías, varías comidas"
Las prácticas actuales, se caracterizan por la disminución en la ingesta de alimentos diarios, la preparación de nuevas comidas, la aparición de nuevas formas de cocinar, la compra de la mayoría de alimentos perecederos y no perecederos, el uso de estufas de gas, el consumo de alimentos económicos, la búsqueda de alimentos en diversas fuentes como subsidios del estado, comedores escolares o comunitarios, y la recolección de alimentos desechos en plazas de mercado, ..." que voy a abastos, yo voy a abastos a recoger verdura, entonces gracias a dios, por ese lado, me ha servido"'... "Yo lo recojo, yo lo recojo, yo no compro nada, gracias a Dios, no compro nada, claro que uno lucha pa, mirar lo que más bueno este"... " Acá he aprendido que no se debe 
echarle tanto condimento a la comida, cocinar bajito de sal, he aprendido todo eso, en poca grasa".

Las nuevas prácticas que emergen como resultado de la adaptación al nuevo estilo de vida, son precarias, puesto que la dieta alimentaria familiar es insuficiente, la cantidad de proteínas y de comidas diarias se disminuye, y la forma de obtener alimentos termina siendo insalubre. Por lo tanto, se puede afirmar que las familias desplazadas generan una mala adaptación a su nuevo entorno, pues no logran satisfacer las necesidades de una dieta balanceada. El reajuste que se realiza en las condiciones de supervivencia, afecta la alimentación, puesto que la economía familiar limita el acceso de alimentos, dando prioridad a otros gastos como lo son los servicios públicos y el arriendo. La alimentación pasa a un segundo plano en la distribución de recursos económicos, por lo que se busca encontrar alimentos de forma gratuita, solicitándolos como regalo o tomándolos de las canecas de basura. El cambio abrupto en la forma de adquirir los alimentos repercute en la forma en que las familias se adaptan, puesto que viviendo en zonas rurales el acceso a alimentos es más factible, en contraste, en las ciudades los ingresos económicos son determinantes para poder acceder a ellos.

\section{Discusión}

Los discursos de las madres de familia abordan los cambios primordiales de la alimentación a partir de la migración forzada, ahondando en las dificultades actuales para la adquisición de alimentos y la modificación y aparición de nuevas prácticas de consumo. Como consecuencia del desplazamiento forzado, las personas al migrar deben adaptarse al entorno, es así como surgen diversas prácticas de consumo. Teniendo en cuenta que la mayoría de las migraciones se originan desde sitios rurales hacia grandes urbes, los cambios en las prácticas culturales y de consumo, se afectan de forma directa.

Inicialmente, es necesario reconocer que la Organización Mundial de la Salud (oms, 2015) establece que una dieta saludable debe estar compuesta por cinco comidas diarias que incluyan proteínas, cereales frutas y verduras. A partir de los hallazgos previos, se identifica que la alimentación actual de las familias carece de alimentos inocuos y nutritivos. Además, las porciones diarias no logran suplir las necesidades dietéticas del organismo, por lo que las víctimas se encuentran en un estado de inseguridad alimentaria.

Por otra parte, los datos planteados en los estudios de Miguel et al. (2012), dan cuenta que las prácticas alimentarias de la población migrante desfavorecen la salud apropiada de los menores de edad, por lo que se podrían desarrollar patologías. Los relatos de las víctimas acreditan que las prácticas de alimentación actual son insanas, principalmente por la carencia de suficientes alimentos nutritivos, es así, como las familias desplazadas se encuentran en riesgo de adquirir enfermedades que perjudican el estado de salud.

En concordancia con los estudios nacionales del Instituto Colombiano de Bienestar Familiar (ICBF, 2010), Gamboa et al. (2007), Machado et al. (2014) y Prada et al. (2008), las narraciones de las madres, expresan como motivo principal de la falta de alimentos, la escases de recursos económicos para adquirirlos. Frente a las altas demandas económicas exigidas por la vida en las ciudades, las familias se ven obligadas a reducir gastos, disminuyendo la compra de alimentos y dando prioridad al pago de servicios públicos, transporte y arriendos.

La ingesta actual de alimentos se destaca por ser insuficiente, las familias en su mayoría, consumen entre una y dos comidas diarias, además, la composición de cada una de estas posee mayoritariamente harinas. Debido a las nuevas formas de adquirir alimentos, en las familias se ha incrementado el consumo de frutas y verduras, ya que estos víveres se obtienen de forma gratuita en los desechos de las plazas de mercado. No obstante, el consumo de proteínas de origen animal es limitado, ya que los costos de dichos alimentos sobrepasan la posibilidad de compra que tienen los consumidores. Lo anterior, acoge las afirmaciones del estudio en el departamento de Santander de Prada, Herrán y Villamizar (2010), en el cual se plantea que las víctimas desplazadas mantienen una dieta alimentaria exigua.

Dentro de lo encontrado en el estudio, es pertinente profundizar en las nuevas prácticas de con- 
sumo adquiridas como resultado de la adaptación al entorno. Una de las prácticas que genera mayor impacto, es la recolección de alimentos en los desechos de plazas de mercado. Dentro del discurso de las mujeres, se infiere que dicha práctica se ha convertido en una constante habitual. Asimismo, esta práctica se hace común en la familia, por lo que asisten al lugar de recogida de alimentos juntos y utilizan elementos característicos de la compra de alimentos "esa misma tarde, salí con mis hijas, y fui, fue una bendición, pues no tenía que darle de comer a mis hijas, y ese día recogimos plátanos que habían sacado"... ' Entonces yo mejor voy con el carrito, me traigo mi verdurita con las peladas, $y$ ellas me acompañan". La carencia de dinero afecta la decisión de compra de alimentos, desde la forma en que se accede a ellos hasta la calidad y cantidad de comidas consumidas, por tanto, surgen prácticas específicas en las familias (Arboleda-Montoya \& Franco-Giraldo, 2012).

El hecho de que las familias se vean obligadas a adquirir alimentos en los desechos, ilustra la ineficacia de las políticas públicas diseñadas para la restitución de derechos. La más reciente, la ley 1448 del 2011 (Ministerio del Interior y de Justicia, 2012) enuncia que se deben contar con medidas de rehabilitación, restitución, indemnización, satisfacción y garantías de no repetición a las víctimas, la ley propone que las familias recibirán ayuda humanitaria inmediata, bajo la cual se suple la ingesta de alimentos. A pesar de que las familias reciben suministros alimentarios, durante los primeros meses de asentamiento en la ciudad, la demanda alimentaria exacerba los recursos brindados por el estado, es así como el derecho humano a la alimentación se ve vulnerado y los jefes o las jefas de hogar generan nuevas prácticas de consumo que les permita asegurar la alimentación familiar. Es innegable que la vulneración del derecho alimentario ocasiona de forma consecuente inseguridad alimentaria, por lo que los hallazgos de Herrán y Patiño (2015) se reafirman al determinar que las familias desplazadas no poseen un goce efectivo del derecho a la alimentación.

Los cambios en las prácticas de consumo alimentario son absolutas, por lo que la modificación en el estilo de vida de los migrantes se ve deteriorado, es- to con cuerda con los planteamientos de otros estudios (Aysa-Lastra, 2011; Bozzoli et al., 2012; Carrillo, 2009; Espinosa-Menéndez et al., 2012; Ibáñez \& Moya, 2006; Macuacé, 2009; Muggah, 2000).

A modo de conclusión, el informe del Programa Mundial de Alimentos (PMA, 2005), se complementa con la información encontrada en la presente investigación, ya que al igual que en los resultados del PMA, en lo reportado por las madres de familia, es público el hecho de que se han reducido el número de meriendas diarias. De acuerdo con el análisis de las narraciones de las madres a cargo de la alimentación, se puede concluir principalmente, que el hecho victimizante del desplazamiento forzado modifica las prácticas de consumo alimentario, ya que las familias se deben adecuar a un estilo de vida en la ciudad, en dónde los alimentos deben adquirirse por medios económicos. Debido a la ineficacia de las políticas públicas y la carencia de acceso a medios monetarios, las familias incursionan en nuevas prácticas de consumo que les permitan reducir gastos, siendo las principales la búsqueda de alimentos en desechos de plazas de mercado; la compra de alimentos no perecederos económicos como el pollo y las menudencias; la reducción de las comidas diarias, pasando de tres a dos, o inclusive a una; la búsqueda de subsidios escolares que garanticen la alimentación de los menores de edad; la incursión a nuevos alimentos y la variedad de preparación de los mismos.

Finalmente, los hallazgos del estudio hacen un llamado a las entidades públicas a cargo de la alimentación y de la restitución de derechos de las víctimas, puesto que son ellas las encargadas de intervenir en las falencias de seguridad alimentaria que se están presentando en las familias víctimas del conflicto actualmente. Se sugiere profundizar en el desarrollo de investigaciones que permitan conocer de manera detallada los estados de nutrición de las familias, haciendo uso de medidas antropométricas para conocer posibles desórdenes alimentarios como el bajo peso, la desnutrición y obesidad. Además, para futuros estudios relacionados, se recomienda recolectar información en diversas ciudades, con el fin de conocer qué otro tipo de adaptaciones realizan las familias para sobrevivir en las grandes urbes y determinar si existe alguna comunalidad entre ellas. 


\section{Referencias}

Arboleda-Montoya, L.M. \& Franco-Giraldo, F.A. (2012). Significado de la alimentación y complemento alimentario mana en un grupo de hogares de Turbo, Colombia. Perspectivas en Nutrición Humana, 14(2). 171-183

Aysa-Lastra, M. (2011). Integration of Internally Displaced Persons in Urban Labour Markets: A Case Study of the IDP Population in Soacha, Colombia. Journal of Refugee Studies, 24(2), 277-303. http://doi.org/10.1093/jrs/feq054.

Bozzoli, C., Bruck, T., \& Wald, N. (2012). Selfemployment and Conflict in Colombia. Journal of Conflict Resolution, 57(1), 117-142. http:// doi.org/10.1177/0022002712464849.

Carmona, J. (2011). Alimentación y estado nutricional de los niños en zonas palúdicas de Antioquia (Colombia). Revista Medunab, 14(2), 94-102. Universidad Autónoma de Bucaramanga.

CARR Center for Human Rights Policy \& Harvard Humanitarian Initiative (2015). Evaluation of Integral Reparations Measures in Colombia. Recuperado de: http://pdf.usaid.gov/pdf_docs/ PA00KSXQ.pdf.

Carrillo, A. C. (2009) 'Internal displacement in Colombia: humanitarian, economic and social consequences in urban settings and current challenges', International Review of the Red Cross, 91(875), 527-546. http://doi.org/10.1017/ S1816383109990427.

Centro Nacional de Memoria Histórica (2013). Basta Ya: Colombia, memorias de guerra y dignidad. Informe general del grupo de memoria histórica. Recuperado de: http://www. centrodememoriahistorica.gov.co/descargas/ informes2013/bastaYa/basta-ya-colombiamemorias-de-guerra-y-dignidad-2016.pdf.

Consultoría para los Derechos Humanos y el Desplazamiento [CODHES]. (2014). Desplazamiento forzado intraurbano y soluciones duraderas Vol. 2 Bogotá, Cúcuta y Quibdó. Recuperado de: http://www.acnur.org/t3/ uploads/media/9609.pdf.
Consultoría para los Derechos Humanos y el Desplazamiento [CODHES], Boletín informativo. (2016). Informe sobre desplazamiento forzado masivo múltiple en Colombia. Recuperado de: http://www.codhes.org/index. php?option=com_si\&type $=4$.

Custodio, D., Ortiz, G., \& Rodríguez, F. (2014). Alimentación, actividad física y otros factores de riesgo cardio-metabólico en la población inmigrante en España. Revisión Bibliográfica. Revista Española de Salud Pública, 88(6), 745 -754.

Espinosa Menéndez, N., Galindo Rosero, A., Bastidas Lopera, W., \& Monsalve Rojas, J. E. (2012). Estado actual de la población víctima de desplazamiento forzado en cuanto al enfoque diferencial de su atención, el derecho a la integridad, la subsistencia mínima y al ingreso económico. El Ágora Universidad San Buenaventura, 12(1), 19-46.

Gamboa, E., López, N., Vera, L., \& Prada, G. (2007). Patrón alimentario y estado nutricional en niños desplazados en Piedecuesta, Colombia. Revista de salud Pública, 9(1), 129-139.

Gómez, L., Ibarra, M., Lucumi, D., Arango, C., Parra, A... Parra, D. (2012). Alimentación no saludable, inactividad física y obesidad en la población infantil colombiana: un llamado urgente al estado y la sociedad civil para emprender acciones efectivas. Global Health Promotion, 19(3), 87-92.

Hernández Bello, A. \& Gutiérrez-Bonilla, M. (2008). Vulnerabilidad y exclusión: Condiciones de vida, situación de salud y acceso a servicios de salud de la población desplazada por la violencia asentada en Bogotá - Colombia, 2005. Revista Gerencia y Políticas de Salud, 7(14), 145-176.

Herrán, O. \& Patiño, A. (2015). Derecho a la alimentación en población en situación de desplazamiento forzado en Bucaramanga, Colombia. Revista Universidad y Salud, 17(2), 192-200.

Ibáñez, A. M. \& Moya, A. (2006). ¿Cómo el desplazamiento forzado deteriora el bienestar de los 
hogares desplazados?: análisis y determinantes del bienestar en los municipios de recepción. Bogotá. Recuperado de https://economia. uniandes.edu.co/components/com_booklibrary/ebooks/d2006-26.pdf

Instituto Colombiano de Bienestar Familiar [ICBF] (2010). Encuesta Nacional de la Situación Nutricional en Colombia. Recuperado de: http://www.icbf.gov.co/portal/page/portal/ Descargas1/Resumenfi.pdf

Internal Displacement Monotoring Center \& Norwegian Refugee Council (IDMC) (2016). Global report on internal displacement. Recuperado de: http://www.internal-displacement.org/ assets/publications/2016/2016-global-reportinternal-displacement-IDMC.pdf

Internal Displacement Monotoring Center \& Norwegian Refugee Council (IDMC) (2017). Global report of internal displacement. Recuperado de: http://www.internal-displacement.org/ global-report/grid2017/pdfs/2017-GRID.pdf

Lakshman, R. (2011). Impact of Displacement on Women and Female-headed Households: A Mixed Method Analysis with a Microeconomic Touch. Journal of Refugee Studies, 26(1), 26-46.

Macuacé, R. (2009). Una aproximación socioeconómica a los impactos del fenómeno del desplazamiento forzado en los municipios del Tambo y Timbio. Biotecnología en el Sector Agropecuario y Agroindustrial, 7(1), 91-101.

Machado, M., Calderón, V., \& Machado, J. (2014). Determinantes socioeconómicos, inseguridad alimentaria y desnutrición crónica en población desplazada de primera infancia, Pereira, Colombia. Revista Médica de Risaralda, 20(1), 3-8.

Marín, A., Gutiérrez, J., Guallar, P., Banegas, J., Regidor, E., \& Rodríguez, F. (2010). Prevalencia de obesidad en inmigrantes en Madrid. Medicina Clínica, 134(11), 483-485.

Menal, S., Fajo, M., \& Marques, I. (2011). Estudio descriptivo del desayuno en una población infantil inmigrante escolarizada, 2007-2010. Revista Española de Nutrición Humana y Dietética, 15 (4), 177-183.

Miguel, B., Cruz, C., Masvidal, R., Frutos, E., Estabanell, A., \& Riera, D. (2012). Alimentación en población infantil inmigrante recién llegada. Enfermería Clínica, 22(3), 118-125.

Ministerio del Interior y de Justicia (2012). Ley de víctimas y Restitución de Tierras. Recuperado de: https://www.centrodememoriahistorica. gov.co/micrositios/caminosParaLaMemoria/ descargables/ley1448.pdf

Mogollón, A. \& Vázquez, M. (2006). Opinión de las mujeres desplazadas sobre la repercusión en su salud del desplazamiento forzado. Gaceta Sanitaria, 20(4), 260-265.

Muggah, R. (2000). Through the developmentalist's looking glass: Conflict-induced displacement and involuntary resettlement in Colombia. Journal of Refugee Studies, 13(2), 133-164.

Oliván, G. (2000). Evaluación del estado de salud y nutrición de los adolescentes inmigrantes ilegales de origen magrebí. Anales Españoles de Pediatría, 53(1), 17-20.

Oliván, G. (2004). Diferencias en el estado de nutrición y salud entre adolescentes inmigrantes ilegales de Marruecos y Argelia. Medicina Clínica, 122(10), 372-374.

Organización Mundial de la Salud (2015). Alimentación Sana: nota descriptiva número 394. Recuperado de: http://www.who.int/ mediacentre/factsheets/fs394/es/

Pareja, A. \& lañez, A. (2014). Violencia contra la mujer y desplazamiento forzado. Análisis de las estrategias de vida de jefas de hogar en Medellín. Acta Sociológica, 65, 151-171.

Prada, G., Herrán, O., \& Ortiz, R. (2008). Patrón alimentario y acceso a los alimentos en familias desplazadas en el municipio de Girón, Santander, Colombia. Revista Panamericana de Salud Pública, 23(4), 257-263. 
Prada, G., Herrán, O., \& Villamizar, I. (2010). Caracterización del consumo de alimentos en familias desplazadas del municipio de Lebrija, Santander. Colombia 2006. Revista Universidad Industrial de Santander Salud, 42 (1), 18-24.

Programa Mundial de Alimentos (2005). Estado nutricional, de alimentación y condiciones de salud de la población desplazada por la violencia en seis subregiones de Colombia. Recuperado de: http://documents.wfp.org/stellent/groups/ public/documents/liaison_offices/wfp086486. pdf?_ga=1.160830973.151807018.1477349770
Registro Único de Víctimas (2017). Reporte de Víctimas del conflicto armado. Recuperado de: http://rni.unidadvictimas.gov.co/RUV

Twinning, P. \& Heller, S.R. (2017). Some guidance on conducting and reporting qualitative studies. Computers \& Education, V. 106, A1-A9. http:// dx.doi.org/10.1016/j.compedu.2016.12.002

United Nations Office for the Coordination of Humanitarian Affairs (OCHA) (2015). Humanitarian Needs Overview 2016. Recuperado de: http://reliefweb.int/sites/reliefweb.int/files/resources/2016_hno_syrian_ arab_republic.pdf 